\title{
Efectos citotoxicos in vitro de extractos y fracciones de Espeletia killipii Cuatr. frente a lineas celulares tumorales humanos
}

\author{
Alba N. Téllez Alfonso ${ }^{1 *}$, Clemencia de Castro², Tulia Riveros de Murcia ${ }^{2}$, \\ Rubén Torrenegra ${ }^{1}$
}

${ }^{1}$ Departamento de Química, Universidad Javeriana, Grupo de Investigación Fitoquímica, Bogotá, Colombia, ${ }^{2}$ Instituto Nacional de Cancerología E.S.E., Grupo de Investigación Nuevos Fármacos en Oncología, Bogotá, Colombia

\begin{abstract}
RESUMO: "Efeitos citotóxicos in vitro de extratos e frações de Espeletia killipii Cuatr. frente a linhas celulares tumorais humanas". O extrato etanólico e as frações de Espeletia killipii (espécie endêmica da vegetação dos páramos do altiplano Cundiboyacense); mostraram atividade citotóxica significativa in vitro nas linhagens celulares tumorais humanas de câncer de mama MCF-7, CSC1170, CSC-1595, CSC-3322, CSC-3325 e na linhagem Hep-2 de laringe. A fração $\mathrm{CH}_{2} \mathrm{Cl}_{2}$ e suas sub-frações foram ativas contra as linhagens celulares cancerígenas de mama na concentração de $50 \mu \mathrm{g} / \mathrm{mL}$, obtendo-se percentagens de viabilidade entre 13 e $20 \%$. O principio ativo ainda não identificado foi obtido por ensaios bioguiados sucessivos e apresentou valores de Concentração Citotóxica media $\left(\mathrm{CC}_{50}\right)$ menores que $1 \mu \mathrm{g} / \mathrm{mL}$ para as linhagens celulares colombianas $\mathrm{CSC}-1170$, CSC-1595, CSC-3322 e CSC-3325; CC50 = $1 \mu \mathrm{g} / \mathrm{mL}$ contra MDA MB 435 e NCl-H23; contra MCF-7 uma $\mathrm{CC}_{50}=2 \mu \mathrm{g} / \mathrm{mL}$ e uma $\mathrm{CC}_{50}$ superior a $16 \mu \mathrm{g} / \mathrm{mL}$ contra PC-3 e U-251.
\end{abstract}

Unitermos: Espeletia killipii, Asteraceae, atividade citotóxica.

\begin{abstract}
In vitro cytotoxic effects of extract and fractions of Espeletia killipii Cuatr. against human tumor cell lines". It was found that the ethanol extracts and fractions of Espeletia killipii (an endemic species of the páramo vegetation of the Cundiboyacense plateau) exhibited cytotoxic activity against several human tumor cell lines. Thus, the extracts and fractions exhibited significant cytotoxic activity against both the human tumor cell lines of breast cancer MCF-7, CSC1170, CSC-1595, CSC-3322, CSC-3325 and the Hep-2 cell lines of laryns. The $\mathrm{CH}_{2} \mathrm{Cl}_{2}$ fraction and its sub-fractions were active against the breast lines at concentration of $50 \mu \mathrm{g} / \mathrm{mL}$, with a viability percentage between 13 and $20 \%$. The active principle, not identified yet, was obtained by successive bio-directed assays. It showed activity against the Colombian cell lines CSC-1170, CSC-1595, CSC-3322 and CSC-3325 at a half Cytotoxic Concentration $\left(\mathrm{CC}_{50}\right)$ less than $1 \mu \mathrm{g} / \mathrm{mL}$, against MDA MB-435 and NCI-H23 at $\mathrm{CC}_{50}=1 \mu \mathrm{g} / \mathrm{mL}$ against MCF-7 at $\mathrm{CC}_{50}=2 \mu \mathrm{g} / \mathrm{mL}$, and against PC-3 and $\mathrm{U}-251$ at $\mathrm{CC}_{50}$ greater than $16 \mu \mathrm{g} / \mathrm{mL}$.
\end{abstract}

Keywords: Espeletia killipii, Asteraceae, cytotoxic activity

\section{INTRODUCCION}

Como parte del programa de investigación "La Biodiversidad Colombiana como fuente de nuevos fármacos en oncología" se evalúo el efecto citotóxico de los extractos y fracciones obtenidas de especies vegetales, lo cual constituye una etapa primordial en el tamizaje de sustancias promisorias para el tratamiento del cáncer.

Este estudio se dirigió principalmente hacia el establecimiento del potencial de extractos y fracciones obtenidos de la especie Espeletia killipii, Subtribu Espeletiinae (Asteraceae), como fuente de sustancias con actividad citotóxica. E. killipii se caracteriza por su riqueza en metabolitos secundarios, el Grupo de investigación Fitoquímica de la Pontificia Universidad Javeriana ha aislado de Espeletia killipii diferentes sustancias entre ellas diterpenos derivados del kaureno, triterpenos del tipo cicloartano, sesquiterpenlactonas y flavonoides (Torrenegra et al., 1994; 1996). La literatura universal reporta efectos citotóxicos y antitumorales para estos tipos de compuestos, lo cual motivó su inclusión en el programa (Stitcher., 1977, Majekodunmi et al., 1996; Smith-Kielland et al., 1996; Ribeiro et al., 2002).

El efecto citotóxico se determinó por medio del ensayo de viabilidad celular con el método MTT (Bromuro de 3-(4,5-dimetiltiazol-2-ilo)-2,5-difeniltetrazolio).

\section{MATERIAL Y METODOS}

\section{Material vegetal}

Las hojas de Espeletia killipii, se recolectaron en el páramo de Guasca. Un ejemplar se depositó en el Herbario Nacional de Colombia bajo el No. Col. 374925 . 


\section{Extracción y aislamiento}

El extracto etanólico de hojas secas y pulverizadas de E. killipii (32 g), se filtró y concentró, resuspendiendo el residuo seco en $\mathrm{MeOH}$ agua. El extracto floculado (19 g) se le realizó fraccionamiento líquido-líquido con petrol, $\mathrm{CH}_{2} \mathrm{Cl}_{2}$ y AcOEt. La fracción activa $\mathrm{CH}_{2} \mathrm{Cl}_{2}(6.5 \mathrm{~g})$ se separó por cromatografía en columna con la fase RP18 en una relación fase estacionaria: muestra $(1: 30)$ y fue eluída con mezclas MeOH: $\mathrm{H}_{2} \mathrm{O}(8: 2,9: 1 \mathrm{MeOH})$; de la cual se obtuvieron 10 subfracciones denominadas K1 $\mathrm{K} 10$, así: con el eluente $\mathrm{MeOH}: \mathrm{H}_{2} \mathrm{O}(8: 2)$ se obtuvieron K1 (3.2 g), K2 (619.3 mg), K3 (377.7 mg), K4 (549.3 $\mathrm{mg})$; en MeOH:H_O (9:1) K5 (467.9 mg), K6 (428.6 mg), K7 (303 mg); en MeOH K8 (353.8 mg) y K9 (201.4 mg) y en $\mathrm{MeOH}: \mathrm{CH}_{2} \mathrm{Cl}_{2} \mathrm{~K} 10$ (81.5 mg). La subfracción activa K1 (3.2 g), se le realizó separación cromatográfica en columna con fase RP-18 en una relación fase estacionaria: muestra (1:60) fue eluída con $\mathrm{MeOH}: \mathrm{H}_{2} \mathrm{O}$ de la cual se obtuvo 12 subfracciones denominadas K1(1) - K1(12), así: con el eluente $\mathrm{MeOH}: \mathrm{H}_{2} \mathrm{O}$ (6:4) se obtuvieron las subfracciones K1(1) a K1(9). Según los resultados de la actividad y la similitud cromatográfica en CCD Si-gel (Petrol:AcOEt 7:3) se mezclaron las fracciones K1(3) a K1(6); obteniéndose $1.1 \mathrm{~g}$ de esta nueva subfracción denominada K1(3) se separó por CC con RP-18 en una relación fase estacionaria: muestra (1:200) de la cual se obtuvieron 4 fracciones así: en $\mathrm{MeOH}: \mathrm{H}_{2} \mathrm{O}$ (1:1) la fracción 1 (386 mg), MeOH: $\mathrm{H}_{2} \mathrm{O}$ (6:4) la fracción 2 (264 $\mathrm{mg}$ ), en $\mathrm{MeOH}: \mathrm{H}_{2} \mathrm{O}(8: 2)$ la fracción 3 (24.1 mg) y en MeOH: $\mathrm{H}_{2} \mathrm{O}$ (9:1) la fracción 4 (306 mg). De la fracción 1 y 2 precipitó una sustancia $(60 \mathrm{mg})$, la cual fue purificada por cristalización fraccionada con $\mathrm{CHCl}_{3}-\mathrm{MeOH}$ y que mostró la mayor actividad citotóxica.

\section{Cultivos celulares}

Se utilizaron las líneas celulares neoplásicas suministradas por el Instituto Nacional del Cáncer en EEUU: HEP-2 (laringe), CMF-7, MDA MB 435 (seno), NCI H-23 (pulmón), U-251 (sistema nervioso central), PC-3 (próstata) y cuatro líneas de cáncer de seno obtenidas y caracterizadas en el Instituto Nacional de Cáncer de Colombia, denominadas CSC-1170, CSC1595, CSC-3322 y CSC-3325 las cuales presentan las siguientes características:

CSC 1170. Línea celular colombiana obtenida en el Laboratorio de Biología Experimental INC del tumor de una paciente de 55 años con cáncer de seno (ductal infiltrante) estadio III B, con las siguientes características: aneuploide, 68 cromosomas, hormonodependiente sensible a las antraciclinas y 5- fluoracilo.

CSC 1595. Línea celular colombiana obtenida en el Laboratorio de Biología Experimental del INC del tumor una paciente de 77 años con cáncer de seno (ductal infiltrante) avanzado, con las siguientes características: aneuploide, 70 cromosomas, hormonodependiente, sensible a antraciclinas y 5 - fluoracilo.

CSC 3322. Obtenido por el laboratorio de Biología Experimental del INC, del tumor de una paciente de 48 años con cáncer de seno bilateral (ductal infiltrante) con las siguientes características: células epiteliales con amplificación del oncogén erbB2 y mutación del gen supresor de tumor P53.

CSC 3325. Obtenido por el Laboratorio de Biología Experimental del INC, del tumor de una paciente de 53 años con cáncer de seno (ductal infiltrante), estadio III B, con las siguientes características: células epiteliales hormonodependientes

Los cultivos se realizan en cajas de 96 pozos, en el medio L-15 (SIGMA) suplementado con 10\% de suero fetal bovino (GIBCO) y $100 \mu \mathrm{L} / 10 \mathrm{ml}$ penicilina estreptomicina y neomicina(SIGMA 5.000U, $5 \mathrm{mg}$ estreptomicina y $10 \mathrm{mg}$ neomicina/ $\mathrm{ml}$ ). Las células de las diferentes líneas y cultivos secundarios se resuspenden en el medio de cultivo de tal forma que haya 40.000 células en $100 \mu \mathrm{L}$ por pozo. Los cultivos se incuban a $37^{\circ} \mathrm{C}$ y $5 \%$ de $\mathrm{CO}_{2}$ y $100 \%$ de humedad relativa por 24 horas con el objeto de tener un buen crecimiento celular.

\section{Bioensayos}

Los ensayos citotóxicos se realizaron en el laboratorio de Biología experimental de Instituto Nacional de Cancerología de Bogotá Colombia. La viabilidad celular fue determinada por la técnica del MTT, ensayo que se encuentra ampliamente referenciado en la literatura (Studzinski, 1999). Se tomó como control negativo el solvente DMSO $0.2 \%$ y control positivo doxorubicina a $0.08 \mu \mathrm{g} / \mathrm{mL}$.

\section{Principio activo}

Sólido blanco con Pf: $163-165^{\circ} \mathrm{C}$; Rf: 0.53 en petrol: AcOEt (6:4), sílica gel. Coloración verde azulosa con $\mathrm{CoCl}_{2} / \mathrm{H}_{2} \mathrm{SO}_{4} \cdot[\alpha]_{\mathrm{D}}=-21^{\circ}\left(\mathrm{C}=1.1, \mathrm{CHCl}_{3}\right)$. I.R: (KBr ). $1780 \mathrm{~cm}^{-1}, 1740$ y $1240 \mathrm{~cm}^{-1}$ y en $1720 \mathrm{~cm}^{-1}$. EM: $\mathrm{M}^{+}: \mathrm{m} / \mathrm{z} 448\left(\mathrm{C}_{23} \mathrm{H}_{28} \mathrm{O}_{9}\right) ; 388 ; 348 ; 83,55$ (pico base).

\section{RESULTADOS Y DISCUSIÓN}

A las fracciónes petrol, $\mathrm{CH}_{2} \mathrm{Cl}_{2}, \mathrm{y}$ AcOEt obtenidas a partir del extracto total etanólico de hojas de $E$. killipii, se les evaluó el efecto citotóxico a la concentración de $100 \mu \mathrm{g} / \mathrm{mL}$, frente a las líneas derivadas de cáncer de seno CMF-7, CSC-1170, CSC-1595, CSC-3322 y CSC3325; las cuatro últimas obtenidas y caracterizadas por el Laboratorio de biología experimental del Instituto Nacional de Cáncer en Colombia y la línea HEP-2 de laringe. Se consideraron activas las fracciones que presentaron porcentajes de viabilidad inferiores a un 50 $\%$. La fracción $\mathrm{CH}_{2} \mathrm{Cl}_{2}$ presentó la mayor actividad como se muestra en la Tabla 1; con porcentajes de viabilidad 
inferiores a $44 \%$ siendo más activo para la línea MCF-7 con un $14 \%$ de viabilidad y no activo para la línea Hep-2 de laringe.

La fracción $\mathrm{CH}_{2} \mathrm{Cl}_{2}(6.5 \mathrm{~g})$ se percoló en una columna empacada con RP-18 en una relación fase estacionaria: muestra 1:30 y eluída con mezclas $\mathrm{MeOH}$ :
$\mathrm{H}_{2} \mathrm{O}$, obteniéndose 10 subfracciones denominadas $\mathrm{K} 1$, $\mathrm{K} 2$ - K10, a las cuales se les evaluó su efecto citotóxico en las líneas celulares a una concentración de $100 \mu \mathrm{g} /$ $\mathrm{mL}$, demostrándose actividad en las subfracciones denominadas K1 y K3, como se demuestra en la tabla 2. Teniendo en cuenta que la subfracción K1 fue más activa

Tabla 1. Porcentaje de viabilidad de las células con relación al control (DMSO 0.2\%) para las fracciones Petrol, $\mathrm{CH}_{2} \mathrm{Cl}_{2}$ y $\mathrm{AcOEt}$ frente a las líneas celulares probadas. Concentración aplicada $100 \mu \mathrm{g} / \mathrm{mL}$ para cada una.

\begin{tabular}{cc|c|c}
\hline & \multicolumn{3}{c}{ Porcentaje de Viabilidad } \\
\cline { 2 - 4 } & Fr. Petrol & Fr. CH 2Cl2 & Fr. AcOEt \\
\hline CSC 1595 & 40 & 23 & 89 \\
HEP 2 & 73 & 71 & 70 \\
MCF 7 & 79 & 14 & 96 \\
CSC 3322 & 54 & 21 & 106 \\
CSC 3325 & 85 & 44 & 77 \\
CSC 1170 & 37 & 19 & 90 \\
\hline
\end{tabular}

que K3 por presentar porcentajes de viabilidad con valores inferiores al 36\%, mostrando la mayor actividad sobre la línea MCF-7 (13\%). Esta subfracción presentó actividad para con la línea Hep-2 (36\%).

La subfracción K1 se separó de nuevo por cromatografía en columna con la fase estacionaria RP 18 y las fases móviles $\mathrm{MeOH}: \mathrm{H}_{2} \mathrm{O}$ en orden descendente de polaridad, de ella se obtuvieron 12 subfracciones a las que se les denominó K1(1) - K1(12). La actividad de las mismas a $50 \mu \mathrm{g} / \mathrm{mL}$ se presenta en la tabla 3 . De acuerdo a los resultados las fracciones más activas fueron K1 (3), K1 (4), K1 (5) y K1 (6).

Por similitud cromatografica (CCD, Si gel Petrol: AcOEt 7:3), se mezclaron las fracciones K1(3) a K1(5) para continuar con el fraccionamiento de las mismas por

Tabla 2. Porcentaje de viabilidad de las células con relación al control ( DMSO 0.2\%) para la fracción $\mathrm{CH}_{2} \mathrm{Cl}_{2}$ frente a las líneas celulares probadas. Concentración aplicada $100 \mu \mathrm{g} / \mathrm{mL}$ para cada una.

\begin{tabular}{cccccccccccc}
\hline & \multicolumn{10}{c}{ \% de Viabilidad Celular } \\
\cline { 2 - 32 } & K1 & K2 & K3 & K4 & K5 & K6 & K7 & K8 & K9 & K10 \\
\hline CSC 1595 & 19 & 79 & 23 & 101 & 92 & 96 & 96 & 97 & 96 & 94 \\
CSC 3322 & 20 & 131 & 20 & 88 & 58 & 66 & 63 & 63 & 75 & 91 \\
CSC 3325 & 36 & 80 & 57 & 96 & 98 & 81 & 76 & 77 & 66 & 90 \\
CSC 1170 & 21 & 96 & 26 & 90 & 26 & 70 & 63 & 78 & 106 & 97 \\
HEP 2 & 36 & 84 & 90 & 87 & 89 & 76 & 101 & 72 & 98 & 99 \\
MCF 7 & 13 & 65 & 17 & 61 & 22 & 106 & 106 & 96 & 102 & 97 \\
\hline
\end{tabular}

Tabla 3. Porcentaje de viabilidad de las células con relación al control (DMSO 0.2\%) para la subfracción $\mathrm{K} 1$ de la fracción $\mathrm{CH}_{2} \mathrm{Cl}_{2}$ frente a las líneas celulares probadas. Concentración aplicada $50 \mu \mathrm{g} / \mathrm{mL}$ para cada una.

\begin{tabular}{|c|c|c|c|c|}
\hline & & \multicolumn{3}{|c|}{ PORCENTAJE DE VIABILIDAD } \\
\hline & & $\mathrm{K} 1(10)$ & $\mathrm{K} 1(11)$ & $\mathrm{K} 1(12)$ \\
\hline & & 26 & 50 & 72 \\
\hline & & 33 & 42 & 66 \\
\hline & & 97 & 111 & 97 \\
\hline & & 32 & 42 & 101 \\
\hline & & 23 & 29 & 94 \\
\hline 4 & arma & & & \\
\hline
\end{tabular}


cromatografía en columna con fase RP-18 y la mezcla $\mathrm{MeOH}: \mathrm{H}_{2} \mathrm{O}$ (6:4) de esta separación se obtuvieron 4 fracciones a las cuales se les determinó su efecto citotóxico a $50 \mu \mathrm{g} / \mathrm{mL}$, obteniéndose mayor actividad en las fracciones 1 y 2 como se demuestra en la tabla 4. El porcentaje de viabilidad de estas dos fracciones está por debajo del $30 \%$ para todas las líneas celulares de cáncer de seno evaluadas; incrementándose la actividad para la línea CSC-3325 (13\%).
Figuras 1). En el estudio se mostró cierta especificidad citotóxica frente a las líneas celulares de cáncer de seno, con mayor evidencia en las líneas celulares colombianas CSC- 1170, CSC-1595, CSC-3322 y CSC-3325, en las cuales se encontraron valores de $\mathrm{CC}_{50}$ menores de $1 \mu \mathrm{g} /$ $\mathrm{mL}$; sobre las líneas MDA MB 435 (seno) y NCI H23 (pulmón) se encontró actividad con una $\mathrm{CC}_{50}=1 \mu \mathrm{g} / \mathrm{mL}$; la línea MCF-7 (seno) con $\mathrm{CC}_{50}=2 \mu \mathrm{g} / \mathrm{mL}$; mientras que no se encontró actividad importante para las líneas

Tabla 4. Porcentaje de viabilidad de las células con relación al control ( DMSO 0.2\%) para la subfracción K1(3) frente a las líneas celulares probadas. Concentración aplicada $50 \mu \mathrm{g} / \mathrm{mL}$ para cada una.

\begin{tabular}{ccccc}
\hline & \multicolumn{4}{c}{ PORCENTAJE DE VIABILIDAD } \\
\cline { 2 - 5 } & Fracción 1 & Fracción 2 & Fracción 3 & Fracción 4 \\
\hline MCF 7 & 19 & 19 & 42 & 99 \\
CSC 1170 & 28 & 26 & 35 & 98 \\
CSC 3322 & 19 & 15 & 40 & 90 \\
CSC 3325 & 13 & 12 & 41 & 97 \\
CSC 1595 & 30 & 31 & 39 & 103 \\
\hline
\end{tabular}

De las fracciones 1 y 2 se aisló un compuesto considerado como el principio citotóxico; el cual fue purificado por cristalización fraccionada con $\mathrm{CHCl}_{3}$ : $\mathrm{MeOH}$.

La actividad citotóxica del compuesto activo se evalúo sobre un mayor número de líneas celulares tumorales humanas, NCI-H23 de pulmón, U-251 del sistema nervioso central, PC-3 de próstata y 6 líneas derivadas de cáncer de seno MCF-7, MDA MB 435 concentraciones de 16, 8, 4, 2 y $1 \mu \mathrm{g} / \mathrm{mL}$ (Tabla 5, CSC-1170, CSC-1595, CSC-3322 y CSC-3325, a las

U-251 (SNC) y PC-3 (Próstata) puesto que la actividad presentada fue a una $\mathrm{CC}_{50}$ superior a $16 \mu \mathrm{g} / \mathrm{mL}$.

En el análisis estadístico se mostró un valor de $\mathrm{P}$ entre 1.037 E-12 a 3.06 E-19, lo que indica que los datos de actividad, obtenidos para la sustancia activa, fueron significativamente menores con respecto al control, indicando que el efecto generado por la sustancia es concluyente.

\section{AGRADECIMIENTOS}

Tabla 5. Porcentaje de viabilidad de las células con relación al control ( DMSO 0.2\%) para el principio activo frente a las líneas celulares probadas. Concentracipones aplicadas $16 \mu \mathrm{g} / \mathrm{mL}, 8 \mu \mathrm{g} / \mathrm{mL}, 4 \mu \mathrm{g} / \mathrm{mL}, 2 \mu \mathrm{g} / \mathrm{mL}$ y $1 \mu \mathrm{g} / \mathrm{mL}$ para cada una.

\begin{tabular}{lccccc}
\hline & \multicolumn{5}{c}{ Principio activo Espeletia killipii } \\
\cline { 2 - 6 } \multicolumn{1}{c}{ Líneas celulares } & \multicolumn{5}{c}{ Porcentaje de Viabilidad } \\
\cline { 2 - 6 } & $16 \mu \mathrm{g} / \mathrm{ml}$ & $8 \mu / \mathrm{ml}$ & $4 \mu \mathrm{g} / \mathrm{ml}$ & $2 \mu \mathrm{g} / \mathrm{ml}$ & $1 \mu \mathrm{g} / \mathrm{ml}$ \\
\hline CSC 1170 (Seno) & 14 & 14 & 16 & 28 & 29 \\
HEP 2 (Laringe) & 11 & 12 & 15 & 39 & 41 \\
CSC 3322 (Seno) & 12 & 14 & 15 & 19 & 28 \\
CSC 3325 (Seno) & 13 & 14 & 21 & 24 & 33 \\
CSC 1595 (Seno) & 12 & 19 & 20 & 27 & 83 \\
MCF 7 (Seno) & 14 & 15 & 16 & 29 & 48 \\
MDAMB435 (Seno) & 11 & 11 & 22 & 36 & 50 \\
NCI H23 ( Pulmón) & 38 & 42 & 47 & 48 & 63 \\
U- 251 (S.N.C) & 54 & 56 & 58 & 59 & 86 \\
PC-3 (Próstata) & 55 & 58 & 76 & 78 & Rev. Bras. Farmacogn. \\
\hline & & & & & 15 \\
& & & & & \\
& & & & & \\
\end{tabular}




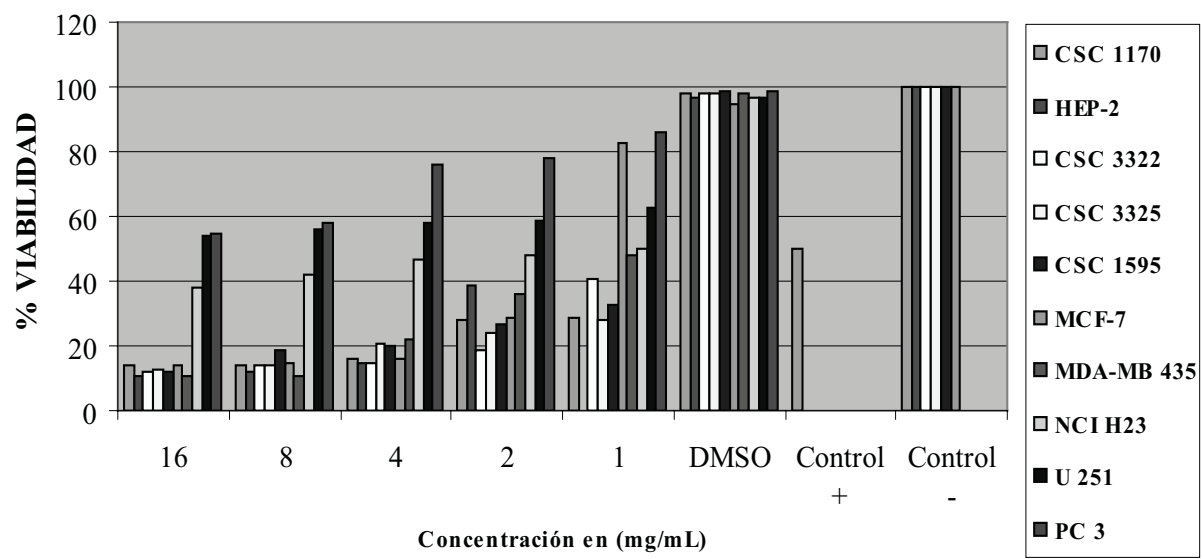

Figura 1. Efecto citotóxico del principio activo

Los autores agradecen a COLCIENCIAS, La Pontificia Universidad Javeriana y el Instituto Nacional de Cancerología por el apoyo financiero. Proyecto "Identificación y efecto de posibles sustancias citotóxicas aisladas de especies vegetales colombianas": Col 120305-11460.

\section{REFERENCIAS}

Majekodunmi O, Fatope M, Oladimeji T, Audu O 1996. Bioactive ent-kaurene diterpenoids from Annona senegalensis. J Nat Prod 59: 301-303.

Ribeiro IM, Silva MTG, Soares RDA, Stutz CM, Bozza M, Tomassini TCB 2002. Physalis angulata L. antineoplasic activity, in vitro, evaluation fromit's stems and fruit capsules. Rev Bras Farmacogn 12(Suppl): 21-23.

Smith-Kielland I, Dornish JM, Malterud K, Hvistendahl G, Romming C, Bockman O, Kolsaker P, Stenstrom Y, Nordal A 1996. Cytotoxic triterpenoids from leaves of Euphorbia pulcherrima. Planta Med 62: 322-325.

Stitcher O 1977. In: New Natural Products and Plant Drugs with pharmacological, Biological or therapeutical Activity (Wagner, H. And Wolff, P., eds). Springer, Berlin.

Studzinski GP 1999. Cell growth, differentiation and senescente. The practical approach. Series editor: B. D. Hames 46-48.

Torrenegra RD, Téllez AN 1994. Química de especies del género Espeletia. E. killipii, E. tunjana. Rev Col Química 23: 29-35.

Torrenegra RD, Téllez AN 1996. Phytochemistry of Espeletia killipii Cuatr. and giberelic activity of some of the isolated compounds. Rev Latinoamer Quím 24: 26. 